Cahiers
de la Recherche
sur les Droits

Cahiers de la recherche sur les droits fondamentaux

15 | 2017

Le corps humain saisi par le droit : entre liberté et propriété

\title{
Contraintes du corps en psychiatrie
}

Constraints of the Body in Psychiatric Care

Françoise Chastang

\section{OpenEdition}

Journals

Édition électronique

URL : https://journals.openedition.org/crdf/558

DOI : $10.4000 /$ crdf.558

ISSN : 2264-1246

Éditeur

Presses universitaires de Caen

Édition imprimée

Date de publication : 1 novembre 2017

Pagination : 107-114

ISBN : 978-2-84133-858-0

ISSN : 1634-8842

Référence électronique

Françoise Chastang, "Contraintes du corps en psychiatrie », Cahiers de la recherche sur les droits fondamentaux [En ligne], 15 | 2017, mis en ligne le 01 octobre 2019, consulté le 15 novembre 2022. URL : http://journals.openedition.org/crdf/558 ; DOI : https://doi.org/10.4000/crdf.558 


\title{
Contraintes du corps en psychiatrie
}

\author{
Françoise CHASTANG \\ Praticien hospitalier au pôle Santé mentale addictologie - département d'accueil et de traitement des urgences du centre hospitalier universitaire (CHU) de Caen \\ Espace de réflexion éthique de Normandie (EREN) \\ Études sur les sciences et les techniques (EA 1610)
}

I. De l'exclusion à la psychiatrie, de la psychiatrie à la santé mentale, de la santé mentale aux valeurs éthiques

A. Un peu d'histoire

B. Place du corps en psychiatrie. La médicalisation de la «Folie»

1. Pinel et le traitement moral

2. Quelques exemples de traitement corporel

3. Contrainte physique, contrainte psychique, contrainte sociale?

II. Une loi spécifique, symbole de la contrainte en psychiatrie

A. La loi du 30 juin 1838 dite "loi sur les aliénés"

B. La transition du $X^{\mathrm{e}}$ siècle : évolution des idées et de la place du patient

C. La loi $n^{\circ}$ 90-527 du 27 juin 1990 relative aux droits et à la protection des personnes hospitalisées en raison de troubles mentaux et à leurs conditions d'hospitalisation

D. La loi $n^{0} 2013-869$ du 27 septembre 2013 modifiant certaines dispositions issues de la loi $n^{0}$ 2011-803 du 5 juillet 2011 relative aux droits et à la protection des personnes faisant l'objet de soins psychiatriques et aux modalités de leur prise en charge

1. La naissance d'une loi

2. Les principes d'une loi

3. Une loi qui définit le cadre sanitaire, administratif et judiciaire des soins en psychiatrie

III. Les grands principes

A. Autonomie et autodétermination

B. De quelle responsabilité parle-t-on?

C. La liberté : un sujet à controverse, l'exemple du suicide

Contraintes du corps en psychiatrie...

Quel vaste sujet, qui nous conduit le long d'une route qui débute avec l'exclusion des fous hors de la société, puis hors de la cité, qui nous fait traverser des chemins bordés d'épines dignes de Vol au-dessus d'un nid de coucou, pour nous faire parvenir aujourd'hui dans une société qui sort la psychiatrie de ses murs, qui intègre le malade mental dans la cité, qui parle de santé mentale plus que de psychiatrie, et qui décline les valeurs de l'éthique comme toute autre spécialité médicale confrontée à la souffrance d'autrui... C'est ce parcours-là que je vous propose de suivre aujourd'hui essentiellement à travers ces deux derniers siècles. 


\section{De l'exclusion à la psychiatrie, de la psychiatrie à la santé mentale, de la santé mentale aux valeurs éthiques ${ }^{1}$}

\section{A. Un peu d'histoire}

Jusqu'à la fin du Moyen Âge, s'est progressivement mis en place un processus de régulation sociale qui contient, marginalise, voire exclut ceux qui incarnent l'idée même d'anormalité. La première forme d'exclusion est l'exclusion familiale: les fous sont chassés, réduits à la mendicité et meurent de faim, car ils sont considérés comme inutiles et incurables. C'est le symbole de la «nefs des fous». À la fin du Moyen Âge, la volonté de normalisation s'accroît et le fou est pris dans la spirale d'enfermement qui touche les mendiants et qui n'épargne pas non plus les autres marginaux.

Le $\mathrm{XVI}^{\mathrm{e}}$ siècle est marqué par l'idéologie de l'enfermement dans toute l'Europe de tous les déviants sociaux, pauvres, libertins, prostituées, vénériens, galeux, mendiants ou insensés. L'édit de 1656 marque le début de ce que Michel Foucault qualifie de «Grand Renfermement » ${ }^{2}$, avec la création d'hôpitaux généraux dans toutes les villes du royaume, assortie d'une logique de travail, les oisifs étant considérés comme insupportables.

Au XVIII ${ }^{e}$ siècle, les maisons de force se multiplient et prennent le relais des hôpitaux généraux. Elles sont au nombre de cinq cents à six cents à la fin du XVIII ${ }^{\mathrm{e}}$ siècle, rattachées à des communautés religieuses. Sur demande de lettre de cachet, signée par de proches parents et adressée au lieutenant de police de Paris, les familles font admettre, moyennant pension, de plus en plus de déviants dans ces maisons. C'est le lieutenant de police qui donne l'ordre ou non d'emprisonner la personne. Au XVIII ${ }^{\mathrm{e}}$ siècle, les insensés sont donc de nouveau pris dans la spirale de l'enfermement qui touche les vagabonds, comme au XVI ${ }^{\mathrm{e}}$ siècle. Et il faudra deux siècles pour sortir de l'amalgame carcéral. Malgré l'apparition, à un certain moment, d'une ébauche de la notion de fou médical, c'est l'image du fou social qui prévaut alors, et la Révolution ne la supprime pas.

$\mathrm{Au}$ lendemain de la Révolution, avec les lois d'août 1790 et de juillet 1791, l'éclatement des communautés religieuses place de nouveau les insensés sous la responsabilité des familles et des communautés d'habitants. Pour la première fois, le «fou » est reconnu comme malade par l'Assemblée constituante du 27 mars 1790, mais il faut attendre 1838 pour qu'il y ait une réponse institutionnelle à la prise en charge des malades mentaux. Pinel, médecin-chef de la Salpêtrière, travaille dès 1791 à la médicalisation de la folie et libère les aliénés de leurs chaînes. En 1818, son élève Esquirol, dans un mémoire pour le ministre de l'Intérieur, esquisse la loi de 1838, propose la création de huit à dix asiles dépendants de l'État, mais financés par les départements. Après quatre projets et de nombreux amendements, la loi est votée à l'unanimité le 22 mars 1838 et signée par Louis-Philippe. Ainsi naît la loi dite « loi sur les aliénés » du 30 juin 1838 qui reste en vigueur jusqu'en 1990. Elle marque la transition entre la période des temps antiques à l'ère moderne dans laquelle l'individu insensé est exclu par la société et la période moderne allant de la période révolutionnaire à contemporaine où la personne reconnue folle est prise en charge par la collectivité. Elle est l'aboutissement de l'abolition des chaînes des aliénés par Pinel, des isolements thérapeutiques d'Esquirol et de l'humanisation des traitements.

\section{B. Place du corps en psychiatrie. La médicalisation de la "Folie »}

"La psychiatrie sans corps, ça n'existe pas», écrit Pierre Delion dans un article intitulé «Franchir le tabou du corps en psychiatrie $»^{3}$.

En libérant les aliénés et en les reconnaissant en tant qu'individus souffrants, Pinel et Esquirol ont accéléré le mouvement de médicalisation de la folie et l'émergence d'une nouvelle spécialité, la psychiatrie. Sont ainsi apparues, après «le traitement moral de la folie», d'autres techniques de soins s'imposant par le biais du corps au patient souffrant de troubles psychiques, dont les plus connues sont les cures de Sakel, les lobotomies, ou les électrochocs, ces derniers demeurant, avec les psychotropes, l'un des traitements phare de l'axe biologique de la psychiatrie actuelle. Mais le corps est également l'un des lieux privilégiés de la souffrance psychique.

\section{Pinel et le traitement moral}

En libérant les aliénés de leurs chaînes, Pinel instaure parallèlement «le traitement moral de la folie», qui consiste à comprendre la logique du délire du patient, puis à s'appuyer sur le reste de raison demeurant chez tout aliéné pour le forcer peu à peu à reconnaître ses erreurs, en usant du dialogue mais aussi, au besoin, de l'autorité médicale. Il recommande de "parler avec douceur», de «compatir à ses maux» et aussi de "donner l'espoir consolant d'un sort plus heureux», tout en maintenant une directivité de type pédagogique et au besoin autoritaire.

Au milieu du XIX ${ }^{\mathrm{e}}$ siècle, ce type de relation a cédé au profit de règles concernant l'organisation et le travail dans les asiles où ont afflué les patients. Par la suite, la liberté dans la relation a constitué une dimension majeure de la prise en soins. Début de dialogue avec la folie pour les uns, ce «traitement moral» fut considéré par d'autres, et

1. F. Granel, Loi no 2011-803 du 5 juillet 2011 réformant les soins psychiatriques sans consentement: impact sur les pratiques cliniques intra-hospitalières dans l'agglomération caennaise pendant les six premiers mois d'application de la loi, thèse d'exercice, médecine, université de Caen Normandie, 2012.

2. M. Foucault, Histoire de la folie à l'âge classique, Paris, Gallimard, 1972.

3. P. Delion, «Franchir le tabou du corps en psychiatrie», L'information psychiatrique, vol. 85, n 1, 2009, p. 24. 
notamment Michel Foucault ${ }^{4}$, comme une autre forme d'aliénation, une autre forme d'exclusion, le malade psychiatrique se trouvant livré au "pouvoir psychiatrique», à la toute-puissance des médecins seuls à juger de la guérison dans leurs asiles.

\section{Quelques exemples de traitement corporel}

La cure de Sakel. Après avoir observé à Vienne en 1933 que les diabétiques se réveillaient brutalement confus et agités après un coma insulinique, Sakel eut alors l'idée de provoquer des comas insuliniques répétés pour traiter les patients atteints de schizophrénie. Le réveil en état de confusion auquel succédait un maternage intensif des soignants permettait à l'équipe d'entrer en contact avec le patient. Les résultats immédiats et intéressants mais temporaires favorisèrent la divulgation de cette méthode qui fut par la suite abandonnée à cause de la confusion intense et persistante et de l'angoisse majeure provoquées.

Les lobotomies. Les lobotomies, qui sont l'un des aspects les plus connus de la psychochirurgie d'après-guerre, consistent en la résection par voie transorbitale de la substance blanche frontale. Ce geste dévastateur conduit à la suppression des fonctions cognitives, voire à des modifications de personnalité. Cette technique, abolie en France dans les années 1970, est dorénavant considérée comme une pratique barbare.

Les électrochocs. L'électroconvulsivothérapie (ECT), anciennement appelée sismothérapie et plus connue sous le nom de traitement par électrochocs, est une méthode utilisée depuis le milieu du $\mathrm{XX}^{\mathrm{e}}$ siècle, et qui consiste à délivrer sous anesthésie générale un courant électrique d'intensité variable sur le cuir chevelu. L'ECT déclenche une crise d'épilepsie de type grand mal. Malgré des résultats appréciables, cette technique fut fort décriée, notamment par le mouvement antipsychiatrique, à cause de ses côtés brutaux, et extrêmement désagréables, et de l'utilisation répressive et punitive qui en a été faite. Néanmoins,

Curieux destin d'une méthode dont les bases théoriques se sont révélées fausses, dont le mode d'action reste mal connu, dont l'indication primitive dans le traitement de la schizophrénie n'est pas bonne, mais qui depuis plus de quarante ans, et en dépit des chimiothérapies modernes, demeure actuellement encore le traitement le plus rapidement efficace de la mélancolie, à laquelle elle n'était pas initialement destinée ${ }^{5}$.

Actuellement, les psychotropes, actifs et de mieux en mieux tolérés, et des approches psychothérapeutiques variées occupent la grande place des thérapies proposées. C'est le développement puis l'essor des psychotropes qui a réellement ouvert le champ de la psychiatrie vers l'espoir d'améliorations spectaculaires et de stabilisation de troubles psychiques. On ne parle plus de «folie», ni même de "maladie mentale», mais de «trouble psychique» ou de «souffrance psychique», et la psychiatrie, en s'ouvrant vers l'extérieur, couvre alors le champ de la santé mentale.

\section{Contrainte physique, contrainte psychique, contrainte sociale?}

Le corps du patient s'exprime dans certaines pathologies, comme les troubles psychosomatiques ou à expression hypocondriaque. Ce peut être également l'expression de la souffrance par les automutilations ou les gestes suicidaires, ou encore de grandes crises d'agitation ou des crises clastiques qui nécessitent alors tout l'arsenal de la contention physique, allant des attaches corporelles aux chambres dites de "soins intensifs» ou de «mise à l'écart». Comme le souligne Pierre Delion, le corps est

[...] le lieu de rencontre et de prise en charge spécifique en fonction de la psychopathologie [...]. [Il] est en quelque sorte l'acteur du sujet, et en tant que tel, il a désormais droit à toutes nos attentions ${ }^{6}$.

Au-delà du corps de l'individu, comment est encore vécue une spécialité comme la psychiatrie? Longtemps exclue, cantonnée en des "asiles » hors de la cité, la psychiatrie s'est progressivement élargie au champ de la «santé mentale», comme si sa dénomination même la cantonnait dans le champ de l'exclusion.

Un métier comme un autre? Ses praticiens n'examinent pas le corps des malades, ce corps qui a de l'importance, car il peut également souffrir, ou faire souffrir par le biais de troubles psychiques, de somatisation. Rappelons que la peau et ses annexes ont la même origine embryonnaire que le système nerveux central, et que nombre de pathologies ont une origine psychosomatique.

Des patients comme les autres? Les patients souffrant de troubles psychiques sont-ils réellement, dans leur vie courante, dans la société, considérés comme des êtres humains comme les autres, ou leur identité n'est-elle pas parfois réduite au trouble psychique? Ce trouble psychique ne représente-t-il pas alors une contrainte psychique, morale?

Un même regard social? Quel regard notre société porte-t-elle réellement sur la personne qui souffre de trouble psychique? Certaines pathologies font encore peur.

Un tabou qui demeure, car, bien que la criminalité des patients psychiatriques ne soit pas supérieure à celle de la population générale, elle peut cependant prendre un aspect brutal, imprévisible, explosif, inhabituel. Ce regard porté sur le patient souffrant de trouble psychique n'est-il pas une contrainte sociale?

4. M. Foucault, Le pouvoir psychiatrique. Cours au Collège de France (1973-1974), Paris, Gallimard - Seuil (Hautes études), 2003.

5. P. Morel, C. Quétel, Médecines de la folie, Paris, Hachette (Pluriel), 1985, p. 123.

6. P. Delion, «Franchir le tabou du corps en psychiatrie», p. 25. 


\section{Une loi spécifique, symbole de la contrainte en psychiatrie ${ }^{7}$}

L'un des symboles actuels de la «contrainte en psychiatrie», qui conduit quasiment de facto les patients vers des hospitalisations en secteurs dits «fermés», est une loi spécifique qui autorise sous conditions les hospitalisations et les soins sous contrainte en psychiatrie.

\section{A. La loi du 30 juin 1838 dite «loi sur les aliénés"}

Conçue par Esquirol, elle stipule dans son article premier que tout département doit être doté d'un établissement public spécialisé à la charge du département pour recevoir et soigner les aliénés. Les premiers établissements sont achevés au milieu du XIX ${ }^{\mathrm{e}}$ siècle, et permettent le désengorgement des prisons, car, en effet, en vertu de l'article 64 du Code pénal de 1810: «Il n’y a ni crime ni délit, lorsque le prévenu était en état de démence au temps de l'action [...]».

À cette époque, l'hospitalisation libre (ou placement libre) n'existait pas, et la loi de 1838 réglementant les hospitalisations en psychiatrie ne distingue que le placement volontaire et le placement d'office. Tous les placements sont consignés dans des registres au sein de chaque établissement. Les établissements peuvent admettre une personne atteinte d'aliénation mentale en placement «volontaire» s'il leur est remis une demande d'admission, avec nom et qualité du demandeur, et un certificat médical très souvent succinct datant de moins de quinze jours et rédigé par un médecin étranger à l'établissement d'accueil et à la famille. Un bulletin d'entrée est envoyé au préfet dans les vingtquatre heures avec un certificat médical d'un médecin de l'établissement qui transmet notification au procureur du roi dans les trois jours. Un nouveau certificat est adressé au préfet dans les quinze jours confirmant ou non le premier. L'article 14 de la loi stipule que toute personne placée au titre du placement volontaire cesse d'être retenue dans l'établissement dès que la sortie sera requise par le curateur, l'époux ou l'épouse, à défaut les ascendants ou les descendants, la personne qui a signé la demande (sauf opposition du conseil de famille). Les placements «d'office», quant à eux, se font sur ordre motivé du préfet, puis un rapport médical, confirmant ou non la mesure, doit lui être adressé chaque semestre. Enfin, la loi prévoit que la personne placée ou retenue puisse saisir, à tout moment, le tribunal qui peut ordonner une sortie immédiate.

Rédigée comme loi de sûreté et d'assistance aux aliénés, la loi de 1838 fut appréhendée comme une loi de contrainte aux soins du fait du développement des asiles, et, dès le début du $\mathrm{XX}^{\mathrm{e}}$ siècle, une réforme de la loi est demandée, avec des arguments à l'appui flous dans lesquels domine essentiellement le risque «d'internement arbitraire».

\section{B. La transition du XXe siècle: évolution des idées et de la place du patient}

L'ensemble du système de santé se développe considérablement dans la seconde moitié du $\mathrm{XX}^{\mathrm{e}}$ siècle. Les lois qui encadrent ce système aboutissent à ce que tout patient ait des droits et à ce qu'il y ait une égalité de droit et une égalité d'accès aux soins entre patients. La Constitution de 1946, puis la Constitution de 1958, sont les premiers textes officiels qui garantissent à chacun un droit à la protection de la santé.

En psychiatrie, les idées évoluent rapidement à la fin du XIX ${ }^{\mathrm{e}}$ et au début du $\mathrm{XX}^{\mathrm{e}}$ siècle. La notion d'aliénation comme affection unique laisse progressivement la place à celle de la maladie mentale selon un modèle médical. Le $\mathrm{XX}^{\mathrm{e}}$ siècle est marqué par la transition de l'asile d'aliénés à l'hôpital psychiatrique. Le terme même d' «hôpital psychiatrique» apparaît suite à la circulaire du 13 octobre 1937, laissant une place aux hospitalisations libres. Il faut attendre 1951 pour que soient créés les services «libres», équivalant aux services ouverts d'aujourd'hui, par opposition aux services «fermés ». La loi n ${ }^{\circ} 81-82$ du 2 février 1981 (dite «loi sécurité et liberté») liste les droits des malades (communication, information, refus de traitement, pratique de la religion de son choix) et leur donne la possibilité de saisir le président du tribunal de grande instance en cas de litige. Dans la continuité de ces travaux, la loi de 1838 sur les aliénés est officiellement réformée le 27 juin 1990.

\section{La loi $n^{0}$ 90-527 du 27 juin 1990 relative aux droits et à la protection des personnes hospitalisées en raison de troubles mentaux et à leurs conditions d'hospitalisation}

Après cent cinquante ans d'application, la loi du 30 juin 1838 est réformée pour donner naissance à la loi du 27 juin 1990 relative aux droits et à la protection des personnes hospitalisées en raison de troubles mentaux et à leurs conditions d'hospitalisation. Parallèlement, en 1991, l'Assemblée générale de l'Organisation des Nations unies (ONU) érige, dans sa résolution $46 / 119^{8}$, les principes internationaux de protection des malades mentaux, renforçant ainsi la recommandation de 1983 du Conseil de l'Europe ${ }^{9}$. Le texte de l'ONU appréhende le consentement comme un consentement éclairé et envisage des situations de dérogation.

7. Voir F. Granel, Loi $n^{\circ}$ 2011-803 du 5 juillet 2011...

8. Assemblée générale de l'ONU, résolution 46/119, «Principes pour la protection des personnes atteintes de maladie mentale et l’amélioration des soins de santé mentale», 17 décembre 1991.

9. Comité des ministres du Conseil de l'Europe, recommandation $\mathrm{n}^{\circ} \mathrm{R}(83) 2$ sur la protection juridique des personnes atteintes de troubles mentaux et placées comme patients involontaires. 
La loi de 1990 affirme et renforce les droits des patients hospitalisés en psychiatrie:

- elle reconnaît officiellement les hospitalisations libres en psychiatrie:

Toute personne hospitalisée avec son consentement pour des troubles mentaux est dite en hospitalisation libre. Elle dispose des mêmes droits liés à l'exercice des libertés individuelles que ceux qui sont reconnus aux malades hospitalisés pour une autre cause ${ }^{10}$.

Les libertés individuelles regroupent le droit à la vie privée, la liberté d'aller et de venir, la liberté de culte, la liberté d'opinion et d'expression;

- la restriction des libertés individuelles doit se limiter à ce qui est nécessaire du fait de l'état du patient;

- elle renforce les droits des patients, avec l'instauration du contrôle des établissements d'accueil par une commission départementale des hospitalisations psychiatriques (CDHP).

Mais, malgré ces avancées, cette loi reste sur le fond très proche de la loi de 1838 , dont elle n'est finalement qu'un toilettage. Elle maintient notamment les deux types d'hospitalisation, à savoir l'hospitalisation à la demande d'un tiers (qui remplace le placement volontaire) et l'hospitalisation d'office (qui remplace le placement d'office), selon des modalités très similaires.

\section{La loi $n^{0} 2013-869$ du 27 septembre 2013 modifiant certaines dispositions issues de la loi $n^{0}$ 2011-803 du 5 juillet 2011 relative aux droits et à la protection des personnes faisant l'objet de soins psychiatriques et aux modalités de leur prise en charge}

\section{La naissance d'une loi}

Trois grands axes ont justifié la modification de la loi de 1990 : la nécessaire harmonisation européenne, la question des droits de l'homme, et l'argument sécuritaire.

Au niveau européen, la France était jusqu'alors le seul pays ne faisant pas intervenir le niveau judiciaire dans ses procédures, et la coexistence d'une procédure «à la demande d'un tiers » et d'une procédure "d'office» par le préfet apparaît bien être une spécificité française.

Par ailleurs, la Cour européenne des droits de l'homme a souligné que les hospitalisations sous contrainte en psychiatrie constituaient une authentique privation de liberté qui se devait d'être encadrée et contrôlée, d'où la nécessaire intervention du juge des libertés.

De plus, pendant l'élaboration de cette loi, deux crimes particulièrement spectaculaires ont été commis en France par des malades mentaux, ce qui a conduit le président de la République française de l'époque, Nicolas Sarkozy, à prononcer un discours dit «d'Anthony» annonçant l'orientation résolument sécuritaire de la nouvelle loi.

\section{Les principes d'une loi}

Elle renforce les droits et garanties accordés aux personnes en soins psychiatriques sans consentement. Son chapitre I est intitulé: «Amélioration de la prise en charge des personnes faisant l'objet de soins psychiatriques sans consentement».

Tout en insistant sur le consentement aux soins et l'autodétermination du patient comme base d'une alliance thérapeutique de qualité, elle autorise sous conditions des soins (et pas uniquement des hospitalisations) sous contrainte, et induit donc une privation de liberté, instaurant ainsi de facto une interaction entre le système judiciaire (par le biais du juge des libertés et de la détention) et le milieu médical. Cette loi donne à ceux qui l'utilisent un pouvoir exorbitant ainsi que des devoirs moraux, éthiques et humains à la hauteur.

Cette loi est fondée sur une éthique déontologique, sur une éthique de la bienfaisance et du soin dirigé vers la personne la plus vulnérable, celle qui ne possède plus, à certains moments de son existence (et non en continu), son libre arbitre ou l'intégralité de ses capacités de raisonnement. Cette loi permet, tout en respectant au maximum la liberté et la dignité, de protéger l'intégrité psychique et parfois même physique de certains patients tels les grands schizophrènes, les grands déprimés, ou les suicidants.

3. Une loi qui définit le cadre sanitaire, administratif et judiciaire des soins en psychiatrie

- Le consentement aux soins, fondé sur le respect de la dignité, des libertés et de l'autodétermination de la personne, demeure la règle et la base d'une relation de soin de qualité; la contrainte doit demeurer l'exception, car elle limite les libertés de la personne.

- La notion de libre choix est inscrite dans la loi et non dans les faits.

- C’est l'absence de soins qui crée un préjudice au patient qui remplit les conditions et non l'inverse; est ainsi réaffirmé le principe de bienfaisance et de déontologie du soin.

- Les soins sous contrainte constituent une dérogation au secret médical.

- Cette loi ne concerne que les personnes souffrant de troubles psychiques pour lesquels les soins hospitaliers, indispensables en fonction des données de la science actuelle, sont refusés, et sans lesquels une mise en danger de soi et / ou d'autrui est à redouter. 
- De telles hospitalisations se déroulent dans des lieux spécifiques habilités.

- Sous le couvert d'instances de contrôle qui sont:

- une instance administrative garante de la procédure, qui est, en fonction de la procédure utilisée, soit le directeur d'hôpital soit le préfet, représentant de l'État;

- une instance judiciaire garante des libertés, à savoir, comme il y a privation des libertés, le juge des libertés et de la détention;

\section{- la CDHP.}

De tels soins, et la loi le mentionne très clairement, ne peuvent se réaliser que dans le plus grand respect des droits des patients, le respect de leur dignité, le respect de leurs libertés en fonction de leur état de santé. Les patients doivent également être tenus informés de leur statut juridique et des modalités de leur hospitalisation.

Ils gardent le droit de communiquer avec leur médecin, un avocat, la CDHP, ou le Contrôleur général des lieux de privation de liberté. Ils peuvent émettre et recevoir du courrier, voter, consulter le règlement intérieur de l'établissement, pratiquer la religion de leur choix et suivre leurs convictions philosophiques.

La mise en route de soins sous contrainte nécessite une évaluation du patient, de son entourage, et de sa capacité à consentir.

L'évaluation du patient comprend la recherche d'un trouble psychique, l'évaluation de sa gravité et l'indication potentielle d'une hospitalisation. Si l'hospitalisation s'avère être d'une absolue nécessité, il convient alors d'analyser la capacité à consentir du patient et l'impact du trouble psychique sur son autodétermination, à savoir sa capacité à recevoir une information adaptée, à comprendre et écouter, à raisonner, à exprimer librement sa décision et à la maintenir dans le temps. Il apparaît alors clairement qu'un patient envahi par des idées délirantes peut avoir du mal à reconnaître son état et à accepter les soins. Un patient en proie à une profonde dépression, isolé dans une gangue glacée qui le coupe de son monde environnant et centré sur une intentionnalité suicidaire intense, refusera les soins. Une telle loi permet donc, dans des cas et dans un contexte bien précis, d'aller au-delà du consentement du patient, et, en reconnaissant l'altération de son psychisme le rendant incapable de prendre une décision librement, permet à l'entourage, avec un certificat médical, de se substituer au patient pour solliciter l'hospitalisation nécessaire.

Dans tous les cas, le patient et son entourage sont informés du caractère pathologique des troubles et de leur retentissement, ainsi que des modalités et des conditions d'application du traitement nécessaire.

La loi définit une pluralité de soins sous contrainte, allant de l'hospitalisation complète aux différents proto- coles de soins, y compris les soins ambulatoires, ce qui en constitue la grande nouveauté. Les soins sont ainsi de plus en plus diversifiés et personnalisés.

Diverses modalités peuvent être utilisées en fonction de la situation présentée et des besoins de la personne:

- les articles L. 3212-1 ou 3212-3 du Code de la santé publique correspondent aux soins à la demande d'un tiers (SDT) (avec le cas particulier de l'admission en péril imminent en l'absence de tiers), lorsque la personne présente un trouble psychique nécessitant impérativement une hospitalisation à laquelle elle ne peut consentir, et lorsque ce trouble met physiquement ou psychiquement la personne elle-même en danger. C'est le cas classique des patients gravement déprimés à haut potentiel suicidaire, ou des patients délirants non dangereux pour autrui. La procédure nécessite la production de deux certificats médicaux détaillés et argumentés, rédigés par deux médecins indépendants dont seul l'un peut dépendre de l'établissement d'accueil, la demande d'un tiers, et le strict respect de la procédure. Des certificats ultérieurs sont nécessaires: celui réalisé dans les vingt-quatre heures confirme ou non la décision d'hospitalisation et le certificat de soixante-douze heures établit les modalités de poursuite des soins;

- l'article L. 3213-1 du Code de la santé publique régit les hospitalisations et les soins sous contrainte sur décision d'un représentant de l'État (SDRE), lorsque la personne présente un trouble psychique nécessitant impérativement une hospitalisation à laquelle elle ne peut consentir, et lorsque ce trouble rend cette personne dangereuse pour autrui. C'est le cas classique des patients délirants hallucinés et persécutés, dangereux pour autrui. Dans cette procédure, les pièces nécessaires à l'admission sont le certificat médical détaillé et argumenté rédigé par un médecin ne dépendant pas de l'hôpital admettant le patient et l'arrêté préfectoral pris au vu du certificat médical. Les certificats ultérieurs sont établis selon les mêmes principes que précédemment.

Dans les deux procédures de contrainte des soins, le juge des libertés intervient uniquement lors des hospitalisations à temps complet dans lesquelles les restrictions de liberté sont les plus notables, dans la première semaine d'hospitalisation, puis dans les six mois lors des hospitalisations prolongées.

Les grandes indications des hospitalisations sous contrainte ont été édictées par la Haute Autorité de santé $^{11}$. Une indication d'hospitalisation ou de soin sous contrainte n'étant pas superposable à un diagnostic clinique, elles dépassent totalement les catégories diagnostiques et concernent plus particulièrement des

11. Haute Autorité de santé, Modalités de prise de décision concernant l'indication en urgence d'une hospitalisation sans consentement d'une personne présentant des troubles mentaux: recommandations pour la pratique clinique, avril 2005, en ligne: https://www.has-sante.fr/portail/upload/docs/ application/pdf/Hospitalisation_sans_consentement_recos.pdf. 
états cliniques, voire des comportements. Sont ainsi concernés:

- le risque suicidaire;

- les troubles de l'humeur;

- les états d’incurie;

- les délires et hallucinations;

- les prises d'alcool et/ou de toxiques.

\section{Les grands principes}

\section{A. Autonomie et autodétermination}

Le concept d'autonomie revêt une pluralité de sens, tel le libre arbitre ( «je fais ce que je veux»), une faculté de discernement («c'est bon pour moi»), ou l'autodétermination ( «c'est mon choix... / j'ai droit à...»), et renvoie à la notion de contrôle et d'hypermaîtrise de l'individu moderne sur sa propre vie. On peut ainsi décrire une autonomie d'action, une autonomie de la volonté, et une autonomie de pensée, cette dernière étant selon Kant la capacité à se «servir de [s] on propre entendement ${ }^{12}$, et par conséquent la capacité de la personne malade à participer à une délibération la concernant. L'autodétermination est considérée comme une faculté rationnelle, comme la liberté de poursuivre ses intérêts et ses préférences sans interférence d'autrui ou de l'État. L'individu alors tout-puissant revendique le droit personnel à évaluer son existence et la volonté de prendre sa vie en main.

En médecine, un acte de soin doit respecter la volonté du patient, dont la liberté est un droit naturel inaliénable. Favoriser l'autonomie suppose donc de fournir une information précise, d'aider à la compréhension par des mots simples et adaptés, afin de permettre à la personne de faire des choix éclairés. C'est le principe du «consentement libre et éclairé», base de toute relation thérapeutique de qualité en médecine.

Une personne est autonome si elle est libre et capable, libre des interférences que pourrait avoir autrui sur ellemême, et capable ou "compétente», c'est-à-dire non entravée par des circonstances physiques, psychologiques ou mentales susceptibles d'invalider son jugement. Or, certaines pathologies psychiatriques, comme les troubles psychotiques et les états dépressifs graves, modifient la perception de la réalité ambiante et les relations à autrui. La personne est temporairement prisonnière de ses propres troubles, et n'est en aucun cas libre de choisir en fonction de ce qu'elle est et de ce qu'elle ferait en temps habituel. Ses propos traduisent alors un état pathologique extrême qui peut être, qui doit être soigné, et non pas un choix «libre et éclairé». Ce sont dans de telles circonstances qu'intervient la loi du 27 septembre 2013 relative aux droits et à la protection des personnes faisant l'objet de soins psychiatriques et aux modalités de leur prise en charge, en permettant soins et assistance à ces personnes particulièrement vulnérables.

\section{B. De quelle responsabilité parle-t-on?}

Selon le dictionnaire, le terme "responsabilité» revêt deux définitions distinctes: soit être cause ou origine d'un dommage, soit l'obligation ou la nécessité morale de répondre ou de se porter garant de ses actions ou de celles des autres. À ces deux définitions correspondent deux angles de vue que sont le lien juridique d'attribution et le lien philosophique de subordination.

Le lien juridique d'attribution correspond à la responsabilité d'une personne devant répondre de quelque chose face à un fait matériel éprouvé, avec un lien logique entre une cause et son effet, entre un auteur potentiel et une action potentiellement blâmable. La personne est donc sanctionnable au regard de la loi, et telle n'est pas aujourd'hui la teneur de notre propos.

Pour le lien philosophique de subordination, le point de départ est pour Levinas la « réponse du sujet à l'appel d'autrui $»^{13}$, et l'engagement que l'on peut avoir en tant que sujet, et encore plus en tant que professionnel de santé, vis-à-vis d'autrui vulnérable: «Nous sommes tous responsables de tout et de tous et moi plus que les autres ${ }^{14}$.

À partir d'un interdit, «Tu ne tueras point», il s'agit donc de répondre à quelqu'un de vulnérable, d'établir la primauté de la relation à l'autre sur la chose, le rôle de l'intersubjectivité, et l'importance du lien de subordination dans cette relation asymétrique, dans cette «articulation entre deux sujets qui n'ont pas la même position, l'un étant du côté de l'appel et l'autre du côté de la réponse ${ }^{15}$.

C'est ainsi que la philosophie de Levinas ${ }^{16}$, fondée sur l'appel d'autrui vulnérable engageant une responsabilité humaine vis-à-vis de l'autre vulnérable et supposant une "passivité» propre à construire une attitude sans jugement, résonne harmonieusement avec la relation de soin telle qu'elle est conçue en psychiatrie. En effet, cette relation de soin est fondée sur la souffrance psychique et les besoins de l'autre, sur sa demande qui engage la véritable responsabilité médicale, à savoir la disponibilité psychique à l'écoute de l'autre en souffrance, base même de la relation et de l'alliance thérapeutique. Et c'est seulement et uniquement au nom de tels concepts et de telles positions que peuvent se justifier les contraintes dans le champ de la santé mentale.

12. E. Kant, Vers la paix perpétuelle. Que signifie s'orienter dans la pensée? Qu'est-ce que les Lumières?, Paris, Flammarion, 2006, p. 43.

13. E. Levinas, Autrement qu'être ou au-delà de l'essence, $2^{\mathrm{e}}$ éd., La Haye, M. Nijhoff, 1978.

14. F. Dostoïevski, Les frères Karamazov, H. Mongault (éd. et trad.), Paris, Gallimard, 1994.

15. E. Levinas, Autrement qu'être...

16. A. Zielinski, Levinas: la responsabilité est sans pourquoi, Paris, PUF, 2004. 


\section{La liberté: un sujet à controverse, l'exemple du suicide}

Plutôt que d'aborder les contraintes et les limitations de liberté dans le cadre des hospitalisations sous contrainte en psychiatrie, permettez-moi d'aborder la liberté sous l'angle de la prévention du suicide, sujet parfois polémique et empreint d'idées reçues provenant de nos réflexions personnelles, éducationnelles, philosophiques ou religieuses.

D’un point de vue légal, le suicide n'est plus sanctionné par la loi, chacun a donc la «liberté» de se suicider, pour autant que ce soit une réelle liberté. Par contre, la loi réprime l'incitation au suicide, mais sans toutefois de positionnement très clair sur l'aide au suicide.

D'un point de vue humain, de quelles libertés parlet-on quand on évoque le droit de mourir? Est-ce d'un droit à «sa » vie, à vivre selon «ses valeurs», ou d'un droit à mourir en fonction ses propres convictions? Est-ce la posture philosophique d'un être en bonne santé hors du désespoir et de la dépression? Plus que revendiquer un «droit à la mort » devant des souffrances psychiques intolérables, la véritable liberté est peut-être celle que l'on s'accorde avec la possibilité de changer d'avis, y compris par le biais de soins lorsque ceux-ci s'avèrent nécessaires.

D'un point de vue médical ${ }^{17}$, il est actuellement bien admis et démontré que, tout en considérant la dimension multifactorielle, $90 \%$ des décès par suicide sont liés à un trouble psychique. Les conduites suicidaires, allant, selon la récente définition de l'Organisation mondiale de la santé (en 2014), des idées suicidaires au geste fatal, sont avant tout caractérisées par un désespoir profond, la recherche de la fin d'une souffrance plus que de la mort physiologique en tant que telle, le tout dans une grande ambivalence, dans une temporalité variable, et dans la recherche d'un lien à l'autre comme nécessaire relation. En d'autres termes, justifier une abstention de soins ou de prise en compte de la souffrance psychique de la personne suicidaire au nom d'une ultime liberté serait aussi stupide que de s'abstenir d'intervenir lors d'un accident de la route parce que le conducteur blessé roulait trop vite et qu'après tout il est en faute, ou de considérer qu'un diabétique en crise ne doit pas être soigné parce qu'il a fait le (mauvais) choix de déguster le gâteau interdit.

En fait, ce serait même pire. Car on peut estimer que le conducteur a fait le «choix» de rouler trop vite, et que le diabétique a fait le «choix» de manger le gâteau en trop. Alors que la personne suicidaire n'a pas fait le choix de souffrir autant, et que le geste suicidaire qu'elle risque alors de réaliser, ou qu'elle a mis en acte, ne résulte pas d'un choix délibéré, mais d'un non-choix, de la seule possibilité qui lui reste pour faire face à sa souffrance, pour échapper à cette douleur psychique intolérable. Dans un tel cas, reconnaître la «liberté de choisir» à une personne engluée dans une souffrance qui ne lui laisse plus aucun choix équivaut à nier cette souffrance, ou du moins à ne pas la reconnaître comme authentique, et humainement à serrer très fort et très vite le nœud coulant de la corde pour se pendre alors que l'on pourrait ôter cette corde, soulager et aider cette personne, et lui redonner vie et goût à la vie. Est-il donc encore possible, dans notre société, au nom du grand principe qu'est la liberté, de ne pas soigner ce qui est (parfaitement bien) traitable, et de ne pas aider qui peut l'être? Car «la liberté de choisir la mort n'est pas plus importante que la liberté de trouver des solutions à nos problèmes et de continuer à vivre $»^{18}$.

La prévention du suicide oppose donc très clairement à un argument libertaire un argument déontologique, fondé sur la conviction qu'il est médicalement nécessaire d'éviter ce qui est évitable, d'aider et de soutenir qui en a besoin, de reconnaitre ce qui peut être curable et de soigner ce qui doit l'être. Comme le souligne Michel Debout, l'un des ardents défenseurs de la prévention du suicide: «Être du côté de la prévention du suicide, ce n'est pas porter atteinte à la liberté d'autrui, c'est simplement lui témoigner que sa vie a de l'importance ${ }^{19}$.

La contrainte du corps, ou par le corps, est une réalité en psychiatrie. C'est même une réalité quotidienne qui n'est jamais une banalité pour qui y est confronté, et, selon les propos d'Emmanuel Hirsch: «C’est acquérir une maturité de médecin que de savoir que certains actes ne sont pas banals ${ }^{20}$. C'est une violence qu'un être humain a le pouvoir d'exercer dans le cadre de sa profession sur un autre être humain, et le professionnel qui agit ainsi doit être en capacité d'expliciter pour quelles raisons humaines, professionnelles et éthiques il fait un tel choix. Chaque acte de contrainte devrait pouvoir être sous-tendu par un bénéfice réel, et permettre de libérer, comme Pinel face aux aliénés, la personne vulnérable de ses temporaires entraves psychiques.

Aussi permettez-moi, en guise de conclusion, de vous laisser méditer les propos suivants d'Albert Camus:

Puisque toute action aujourd'hui débouche sur le meurtre, direct ou indirect, nous ne pouvons pas agir avant de savoir si et pourquoi nous devons donner la mort.

L'important n'est donc pas de remonter à la racine des choses, mais, le monde étant ce qu'il est, de savoir comment s'y conduire ${ }^{21}$.

17. Voir V. Caillard, F. Chastang, Le geste suicidaire, Issy-les-Moulineaux, Elsevier-Masson, 2010.

18. B. L. Mishara, M. Tousignant, Comprendre le suicide, Montréal, Presses de l'université de Montréal, 2004, p. 139.

19. M. Debout, La France du suicide, Paris, Stock, 2002.

20. Cité dans A. Lorriaux, "Médecine des internes à touche-touche avec l'éthique», Libération, 5 juin 2015.

21. A. Camus, L'homme révolté, in Euvres, Paris, Gallimard (Quarto), 2013, p. 848 (nous soulignons). 